\title{
Prevalence and Antibiogram of Acinetobacter Infections: An experience from a Teaching Institute of Rural Setting, in Central India
}

\author{
Aakanksha Sharma, Smita Bawankar* and Mousumi Kilikdar \\ Department of Microbiology, Shri Shankaracharya Institute of Medical Sciences, \\ Junwani, Bhilai, Chhattisgarh, India \\ *Corresponding author
}

\section{A B S T R A C T}

\section{Keywords \\ Acinetobacter, Infection, Antibiotic susceptibility, Multi-drug Resistance, Intensive care unit \\ Article Info \\ Accepted: \\ 12 December 2018 Available Online: 10 January 2019}

\begin{abstract}
Acinetobacter baumannii has emerged as a worldwide problem as a nosocomial pathogen in hospitalized patients. Acinetobacter spp. can cause a multitude of infections including pneumonia, bacteremia, meningitis, urinary tract infections, and skin and soft tissue infections, and the mortality associated with these infections is high. Isolates resistant to almost all commercially available antimicrobials have been identified, thus limiting treatment options. Isolates of Acinetobacter received in the microbiology laboratory over a period of one year were processed, identified by conventional standard methods and antimicrobial susceptibility was performed according to CLSI guidelines. A total of 62 isolates were identified. Maximum $(43.5 \%)$ were from respiratory specimens and indoor patients. Multi drug resistance was observed in $62.9 \%$ isolates. Drug resistance is a major therapeutic concern in Acinetobacter isolates. Even though no pan drug resistant organism was encountered in our study, still judicious antimicrobial use and antimicrobial stewardship program is strongly advocated to curb the growing threat of resistance
\end{abstract}

\section{Introduction}

The gram-negative coccobacillus Acinetobacter, a pathogen once seen only in hot, humid climates, has become an increasingly common nosocomial problem even in temperate climates. (Munoz-Price, 2008) Interest in Acinetobacter spp. has been growing for the past 30 years. One of the main reasons for the present increased interest in this genus is the emergence of multiresistant strains, some of which are panresistant to antibiotics, that suddenly cause an outbreak of infection involving several patients in a clinical unit. (Joly Guillou, 2005) The genus Acinetobacter comprises a complex and heterogeneous group of bacteria, many of which are capable of causing a range of opportunistic, often catheter-related, infections in humans.

In the hospital setting, Acinetobacter species have been implicated in a wide range of infections, particularly in critically-ill patients with impaired host defenses. These infections include pneumonia, skin and soft-tissue infections, wound infections, urinary tract infections, meningitis, and bloodstream 
infections. Nosocomial infections and hospital outbreaks have been attributed mainly to $A$. baumannii, particularly in the intensive care unit (ICU) setting. Acinetobacter spp. have been reported occasionally as causative agents of community-acquired infections such as wound infection, urinary tract infection, otitis media, eye infections, meningitis and endocarditis (Visca, 2011).

Several factors like over the counter antibiotic use, overcrowding in hospitals, imperfect infection control practices, and use of excessive invasive devices contribute to the development of high antimicrobial resistance, especially in developing countries. Additionally, these factors also facilitate easy transmission of Multi drug resistant organisms implicated in various healthcare associated infections (HCAI). (Banerjee $\mathrm{T}$, 2018)

With worldwide reports of increasing isolation of this organism from various samples, we performed retrospective study to estimate the extent of the problem in our teaching hospital and also analyze the prevalent situation for possible control measures.

\section{Materials and Methods}

A retrospective study was conducted in the department of Microbiology, at the teaching institute, over a period of one year (September 2017 to August 2018).

Sample collection: A total of 62 isolates of Acinetobacter species recovered from the urine, pus, blood, respiratory samples such as sputum, endotracheal aspirates, bronchoalveolar lavage (BAL) and high vaginal swabs were included in the study.

For the isolation of Acinetobacter spp., the clinical samples were inoculated onto blood agar and MacConkey agar. After overnight incubation at $37^{\circ} \mathrm{C}$, the suspected colonies were further processed for identification of Acinetobacter species by standard conventional methods. The antimicrobial susceptibility testing of all the 62 Acinetobacter isolates was carried out by Kirby-Bauer disc diffusion method on Mueller-Hinton agar medium and results were interpreted as per the Clinical and Laboratory Standards Institute guidelines. Antimicrobial discs used in the study were procured from Hi-media Laboratories, Mumbai, India. Escherichia coli ATCC 25922 strain was employed as a control

\section{Multi-drug resistant (MDR) Acinetobacter}

Acinetobacter isolates resistant to at least three classes of antimicrobial agents- all penicillins and cephalosporins (including inhibitor combinations), fluoroquinolones and aminoglycosides

\section{Extensively drug resistant (XDR) Acinetobacter}

Acinetobacter isolates resistant to the three classes of antimicrobials described above (MDR) and also resistant to carbapenems

\section{Pan drug resistant (PDR) Acinetobacter}

Acinetobacter isolates resistant to the three classes of antimicrobials described above (MDR), carbapenems, polymyxins and tigecycline.

\section{Results and Discussion}

A total of 62 non-duplicate, non- consecutive Acinetobacter isolates were processed for identification, antimicrobial susceptibility testing was done to know the MDR, XDR and PDR pattern of these isolates. 
The isolation pattern of Acinetobacter from various clinical specimens is depicted in table 1. The higher isolation rates of Acinetobacter from the respiratory specimens is in agreement with literature. Studies on Acinetobacter in various countries have shown a predominance of isolation from urine $(21-27 \%)$ and tracheobronchial secretions (24.8-48.8\%). Genito-urinary tract infections in the form of cystitis and pyelonephritis can be seen in case of indwelling catheters or nephrolithiasis. The organism was responsible for $30.6 \%$ cases of urinary tract infection and $27.5 \%$ cases of wound infection, in a study conducted by Joshi et al., (2006).

The pattern of distribution of Acinetobacter species from various hospital units is reflected in Figure 1. Majority of the isolates were recovered from the patients admitted in wards where a number of risk factors were present, including the fact that patients were hospitalised for very long periods, the moist environment of the catheters/urobags and treatment with antibiotics off and on, all giving an opportunity for the bacilli to colonise various sites and then later turn into a pathogen (Vincent et al., 2009, Lee Sang Oh et al., 2004).

In the present study, Acinetobacter species were found to be resistant to most commonly used antibiotics (Table 2). Resistance towards imipenem and Meropenem was recorded to be
$21 \%$ and $39.35 \%$ respectively. No resistance was seen in Colistin and Polymyxin B in our study which is similar to the study published by Dash et al., and Shareek et al., where all isolates were sensitive to colistin. Out of total isolates $39(62.9 \%)$ were multidrug resistant (MDR) in our study. The other studies conducted by Dash et al., in Odisha and Rekha et al., in Kolar, Karnataka reported MDR isolates to be $55 \%$ and $74 \%$ respectively. Bhattacharya et al., Gupta et al., and Mostofi et al., reported MDR isolates to be $29 \%$; $40 \%$ and $54 \%$ respectively. In ICUs most, sensitive drug was colistin (100\%) followed by imipenem. Acinetobacter appears to have a propensity to develop antibiotic resistance extremely rapidly, perhaps as a consequence of its long term evolutionary exposure to antibiotic producing organisms in soil environment. The emergence of antibiotic resistant strains in ICU is because of higher of use of antimicrobial agents per patient and per surface area.

The antimicrobial susceptibility pattern of the isolate depends on the prevailing epidemiology of the strains circulating in the hospital and community. Thus, regular surveillance and antimicrobial stewardship programs are the need of the hour to promote the judicious use of antibiotics and prevent the development of pan drug resistant strains.

Table.1 Sample-wise distribution of the Acinetobacter isolates

\begin{tabular}{|l|c|}
\hline SAMPLE & NUMBER OF ISOLATES (\%) \\
\hline $\begin{array}{l}\text { Respiratory samples } \\
\text { (Sputum, BAL, Tracheal } \\
\text { aspirates) }\end{array}$ & $27(43.5 \%)$ \\
\hline Pus & \\
\hline Urine & $18(29 \%)$ \\
\hline Blood & $11(17.7 \%)$ \\
\hline High Vaginal Swabs & $3(4.8 \%)$ \\
\hline Others & $2(3.2 \%)$ \\
\hline
\end{tabular}


Table.2 Antibiotic sensitivity pattern of the Acinetobacter isolates

\begin{tabular}{|l|c|}
\hline ANTIBIOTIC & No. of susceptible isolates (\%) \\
\hline Amikacin & $20(32.2 \%)$ \\
\hline Gentamycin & $17(27.4 \%)$ \\
\hline Amoxicillin- Clavulanic acid & $9(14.5 \%)$ \\
\hline Ceftriaxone & $4(6.5 \%)$ \\
\hline Ceftazidime & $3(4.8 \%)$ \\
\hline Cefepime & $8(12.9 \%)$ \\
\hline Ciprofloxacin & $24(38.7 \%)$ \\
\hline Cotrimoxazole & $8(12.9 \%)$ \\
\hline Doxycycline & $10(16.1 \%)$ \\
\hline Imipenem & $49(79 \%)$ \\
\hline Meropenem & $38(61.35)$ \\
\hline Piperacillin/Tazobactam & $48(77.4 \%)$ \\
\hline Colistin & $62(100 \%)$ \\
\hline Polymyxin B & $62(100 \%)$ \\
\hline
\end{tabular}

Figure.1

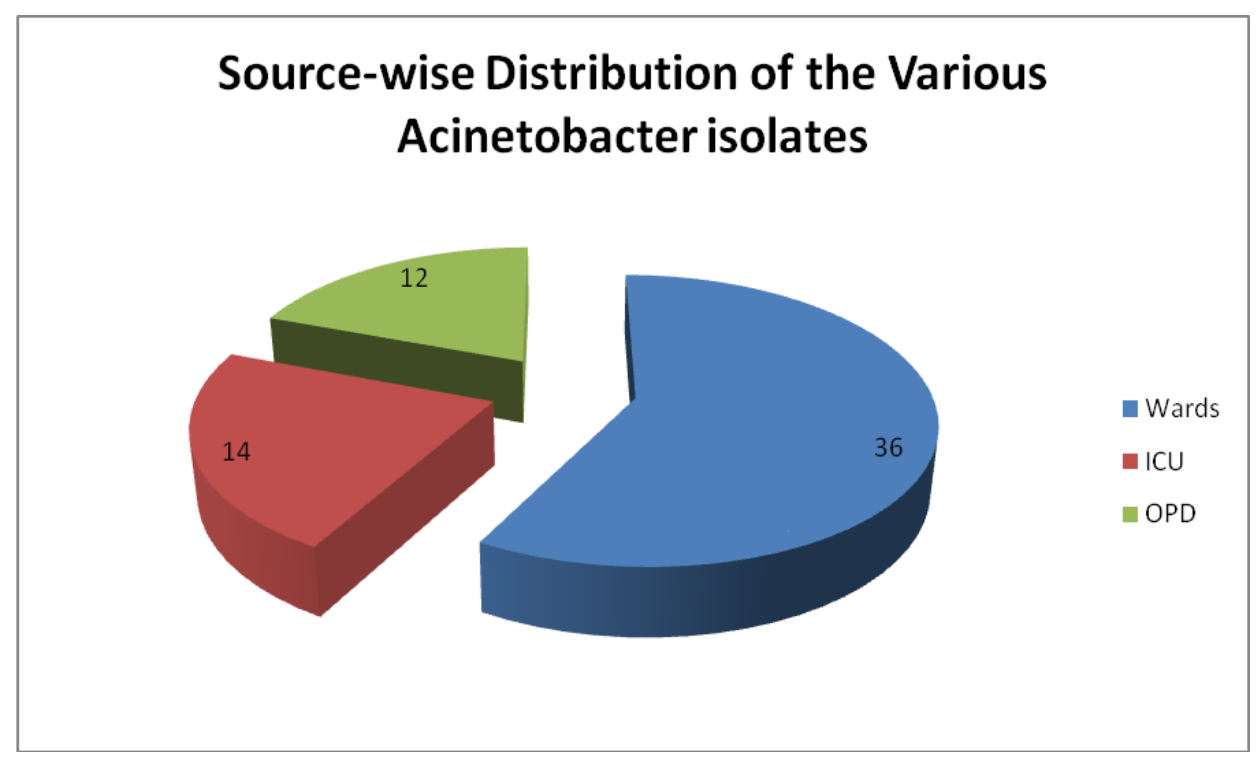

In conclusion Acinetobacter is nowadays a common threat in hospital acquired infections especially in critically ill patients admitted to ICU. Acinetobacter species in our study were found to be resistant to most commonly used antibiotics. It is a great challenge for the physicians to treat MDR Acinetobacter spp. which is independently associated with high mortality, emphasizing the need for aggressive infection control strategies. To avoid resistance, antibiotics should be used judiciously and empirical therapy should be determined for each hospital according to the resistance rates of the hospital. Also since the organism is still susceptible to most of the disinfectants, proper hand hygiene and 
protocol should be maintained to prevent the rise in nosocomial infections.

\section{References}

Banerjee T, Mishra A, Das A, Sharma S, Barman $\mathrm{H}$, and Yadav G, High Prevalence and Endemicity of Multidrug Resistant Acinetobacter spp. in Intensive Care Unit of a Tertiary Care Hospital, Varanasi, India. Journal of Pathogens, vol. 2018, Article ID 9129083, 8 pages, 2018

Bhattacharyya S, Bhattacharyya I, Rit K, Mukhopadhyay PK, Dey JB, Ganguly U, et al., Antibiogram of Acinetobacter spp. isolated from various clinical specimens in a tertiary care hospital, West Bengal, India. Biomed Res. 2013; 24:43-6.

Dash M, Padhi S, Pattnaik S, Mohanty I, Misra P. Frequency, risk factors, and antibiogram of Acinetobacter species isolated from various clinical samples in a tertiary care hospital in Odisha, India. Avicenna J Med [serial online] 2013 [cited 2018 Dec 31];3:97-102.

Gupta N, Gandham N, Jadhav S, Mishra RN. Isolation and identification of Acinetobacter species with special reference to antibiotic resistance. J Nat Sc Biol Med. 2015; 6:159-62.

Joshi SG' Litake GM, Satpute MG, Telang NV, Ghole VS, Niphadkar KB. Clinical and demographic features of infection caused by Acinetobacter species. Indian $\mathrm{J}$ Med Sci. 2006 Sep; 60(9):351-60.

Kamble R. Acinetobacter species in Health Care setting: Clinical significance and Antimicrobial sensitivity Int.J.Curr.Microbiol.App.Sci (2015) 4(4):
861-869

M.-L.Joly-Guillou. Clinical impact and pathogenicity of Acinetobacter. Clinical Microbiology and Infection Volume 11, Issue 11, November 2005, Pages 868-873

Mostofi S, Mirnejad R, Masjedian F. Multi-drug resistance in Acinetobacter baumannii strains isolated from clinical specimens from three hospitals in Tehran-Iran. Afr J Microbiol Res. 2011; 5: 3579-82.

Munoz-Price SL, Weinstein R, Acinetobacter Infection. N Engl J Med 2008; 358:12711281

Saha S, Devi KM, Damrolien S, Devi S. Int J Res Med Sci. 2018 Jun; 6(6):2076-2080

Sang-Oh Lee, Nam Joong Kim, Sang-Ho Choi, Tae Hyong Kim, Jin-Won Chung, JunHee Woo, Jiso Ryu, Yang Soo Kim. Risk Factors for Acquisition of ImipenemResistant Acinetobacter baumannii: a Case-Control Study. antimicrobial Agents and Chemotherapy Feb 2004, 48 (3) 1070.

Upadhyay S, Khyriem AB, Bhattacharya P, Bhattacharjee A, Joshi SR. High-level aminoglycoside resistance in Acinetobacter baumannii recovered from Intensive Care Unit patients in Northeastern India. Indian $\mathbf{J}$ Med Microbiol 2018; 36:43-8

Vincent, Jean-Louis, Jordi R, Marshall J, Eliézer S,Antonio A, Claude DM et al., International Study of the Prevalence and Outcomes of Infection in Intensive Care Units. JAMA 2009; 302(21):2323-9.

Visca P, Seifert H, Kevin J. Towner. Acinetobacter infection - an emerging threat to human health. Life, 63(12):1048-1054

\section{How to cite this article:}

Aakanksha Sharma, Smita Bawankar and Mousumi Kilikdar. 2019. Prevalence and Antibiogram of Acinetobacter Infections: An experience from a Teaching Institute of Rural Setting, in Central India. Int.J.Curr.Microbiol.App.Sci. 8(01): 1674-1678.

doi: https://doi.org/10.20546/ijcmas.2019.801.176 